\title{
Strong deep learning baseline for single lead ECG processing
}

\author{
BOTOS Csaba \\ Pázmány Péter Catholic \\ University \\ 1083 Práter utca 50/A \\ Budapest, Hungary \\ botos.csaba@hallgato.ppke.hu
}

\author{
HAKKEL Tamás \\ Pázmány Péter Catholic \\ University \\ 1083 Práter utca 50/A \\ Budapest, Hungary \\ hakkel.tamas@hallgato.ppke.hu
}

GODA Márton Áron
Pázmány Péter Catholic
University
1083 Práter utca 50/A
Budapest, Hungary
goda.marton.aron@itk.ppke.hu

\author{
HORVÁTH András ${ }^{\ddagger}$ \\ Pázmány Péter Catholic \\ University \\ 1083 Práter utca 50/A \\ Budapest, Hungary \\ horvath.andras@itk.ppke.hu
}

\begin{abstract}
Objective: Atrial fibrillation (AF) is one of the most common serious abnormal heart rhythm conditions, and the number of deaths related to atrial fibrillation has increased by an order of magnitude in the past decades. We aim to create a system, which can provide help for cardiologist, classifying and highlighting important segments in recordings.
\end{abstract}

\begin{abstract}
Approach: In this paper, we propose a novel approach for AF detection using only a deep neural architecture without any traditional feature extractor for real-time automated suggestions of possible cardiac failures that can detect class invariant anomalies in signals recorded by a single channel portable ECG device.
\end{abstract}

Results: Detecting the four categories: Normal, AF, Other and Noisy in terms of the official, F1 metric of hidden dataset maintained by the organizers of PhysioNet Computing in Cardiology Challenge 2017, our proposed algorithm has scored $0.88,0.80,0.69,0.64$ points respectively, and 0.79 on average.

\section{Keywords}

deep learning, residual network, fully convolutional network, time-series, signal processing, ECG, atrial fibrillation, AF detection

\footnotetext{
* Corresponding author.

$\dagger^{\dagger}$ Corresponding author.

$\ddagger$ Corresponding author.
}

\section{INTRODUCTION}

Cardiovascular diseases are responsible for the highest percentage of fatal outcomes among health problems in the modern world. One of the most common and serious abnormal heart rhythm conditions is atrial fibrillation, which affects about $2 \%$ to $3 \%$ of the population in Europe and North America [1]. It is associated with an increased risk of heart failure, dementia, and stroke. Additionally, the number of deaths related to atrial fibrillation has increased by an order of magnitude in recent decades: growing from 29,000 in 1990 up to 193,300 in 2015. Researchers project that by 2030 cardiovascular diseases will account for more than three-quarters of deaths worldwide [2].

While it is essential to develop efficient algorithms to automatize detection for monitoring patients with small portable or wearable devices, and promising methods $[3,4]$ are already available, there is still no completely satisfying solution due to the low signal-to-noise ratio of portable ECG devices, as well as the multiple types and the episodic manner of atrial fibrillation. Unfortunately, detecting atrial fibrillation poses a significant challenge even for the most experienced cardiac exerts. As a result, a larger time window has to be recorded and examined by experts to arrive at a diagnosis.

To promote the solution and draw the attention of the scientific community to this problem, a challenge was introduced by PhysioNet [5], which targets the algorithmic classification of atrial fibrillation signals. In this challenge, 8528 short, single-channel recordings were provided produced by a lowcost, portable device called KardiaMobile, manufactured by AliveCor Inc. [6]. The length of the recordings ranged from 9.0 seconds to 61.0 seconds with an average of 32.5 seconds. These samples were divided into four different classes: atrial fibrillation, normal, noisy signals, and recordings from patients with other cardiac diseases, consisting of 771,5154 , 46 , and 2557 samples, respectively. 


\section{RELATED WORKS}

Using clinically meaningful features, good quality ECG measurements could be flawlessly classified by simply applying traditional machine learning techniques (i.e. logistic regression, random-tree, support-vector-machines). On the other hand, real-life samples often pose too much noise and high variance that could mislead handcrafted rules, and yet stateof-the-art approaches are still relying heavily on feature engineering for $\mathrm{AF}$ detection. Accordingly, three of the four winners of the CinC Cardiology 2017 challenge combined only medically relevant feature-extractors and did not incorporate any neural network-based features $[7,8,9]$.

Only one of the four winner approaches fused expert features with other descriptors extracted by a neural network. Hong et al.[10] proposed an algorithm concerning 64 features learned by a Deep Neural Architecture, namely a timeinvariant hierarchical feature extractor network with 4 residual blocks [11] combined with a Bi-directional Long Shortterm Memory network (LSTM [12]) resulting in a 32 dimensional continuous descriptor and a Uni-directional LSTM trained separately using centerwave input to extract 32 time related features. While the final classifier was applied on a feature space with more than 600 dimensions, after ranking by importance, the top 20 were made up of 17 deep learned features and only the 3 remaining were clinically relevant or external statistical features.

At the same time, many other participants of the Challenge also used neural networks $[13,14,15,16]$ as feature detector in addition to their traditional feature extractors. One of them was Andreotti et al. [17], who compared their featurebased classifiers to residual neural networks. They concluded that their neural networks outperform their featurebased classifiers, showing the strength of the purely neural network-based approach. Parvaneh et al. [18] improved a dense convolutional network by signal quality index and by the transformation of signal to the frequency domain. Their approach was similar to ours as they applied a neural network to extract frequency-domain features. Xiong et al. [19] tried multiple methods with success, which we utilized as well, including skip connections, and a neural network trained on the spectrogram.

\begin{tabular}{lrrrrr}
\hline & Normal & AF & Other & Noise & Avg. \\
\hline Teijeiro et al. & 0.90 & $\mathbf{0 . 8 5}$ & 0.74 & 0.56 & $\mathbf{0 . 8 3}$ \\
Datta et al. & $\mathbf{0 . 9 2}$ & 0.82 & $\mathbf{0 . 7 5}$ & 0.52 & $\mathbf{0 . 8 3}$ \\
Zabihi et al. & 0.91 & 0.84 & 0.73 & 0.50 & $\mathbf{0 . 8 3}$ \\
Hong et al. & 0.91 & 0.81 & $\mathbf{0 . 7 5}$ & 0.57 & $\mathbf{0 . 8 3}$ \\
\hline ours & 0.88 & 0.80 & 0.69 & $\mathbf{0 . 6 4}$ & 0.79
\end{tabular}

Table 1: F1 scores on the hidden test set of the CinC Challenge 2017. The winner algorithms (first 4 rows) excel in different tasks, since they utilize different pools of features. An important note is that in order to reduce prediction uncertainty many have submitted ensembles which improve the overall accuracy; however, does not reveal the true generalizing capabilities of the underlying algorithm.

\section{METHODS}

\subsection{Extending Dataset with Alternative Anno- tation}

We compete with human performance; however, we do not know much about that. There is no better reference currently than human annotation (possibly by experts). Atrial fibrillation is a human category, there is no mathematically exact definition for it, thus we need humans to define its characteristics. Unfortunately, these definitions are vague and fuzzy from the algorithmic point of view as there is always an inherent ambiguity in all applications when we try to approximate human definitions using mathematical models. To come around this problem we have created a dataset in which every sample was annotated by multiple (in our case two) experts to allow us measuring the variation of the annotations as well.

We asked two doctors to help us: Dr Sz. Herczeg PhD student in the field of cardiac arrhythmia (Expert-1), and Dr I. Osztheimer cardiologist consultant (Expert-2), both working at the Heart and Vascular Center of Semmelweis University, in Budapest. Our goal was to examine the difference between the decisions of experts of the Challenge, our doctors, and a model trained on this dataset. By this, we aimed to have an approximation of the accuracy of human performance. Then we wanted to explore which features are the most important ones our model is looking for. Finally, we made some efforts to highlight these important features to help human specialists.

To solve that task, we developed a website that displays the recordings and provides a graphical user interface to annotate the currently displayed recording. Asking our doctors to use that website, we obtained an alternative annotation that helped us to validate the data set, i.e. which the obvious cases are and which samples are too ambiguous to make a clear diagnosis. The website picks recordings randomly, selecting recordings from the four different classes uniformly.

\subsection{Neural Network Architecture}

Based on empirical evidence in the field of computer vision, to reduce training time and to make the resulting detector more robust, we applied recently published methods such as ADAM[20], SELU[21], dilated convolutions [22], residual blocks[23] - for which we will provide a quick overview in this section, and a more detailed description and summary of resulting improvements in appendix A. While several image recognition baseline NN architectures (such as ResNet and VGG) could be re-designed to fit the AF detection task, we developed domain-specific ensembles from core building blocks of the aforementioned baseline architectures. Alongside with the proposed networks, we have applied preand post-processing steps: forked feature extraction on both temporal and spectral domain, and merging encoded feature vectors from different domains directly under the final classifier layer.

Despite the moderate improvements on the temporal and spectral domains by the application of the advanced building blocks (Figure 1), the extension of the logistic regression on multi-domain feature representations resulted in an architecture that could significantly outperform the most robust 


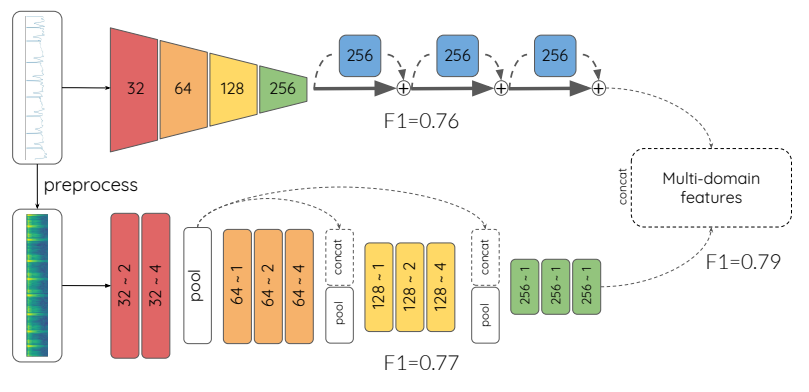

Figure 1: Learning representations from raw timeseries and equal length spectrogram.

networks on individual domains. We performed hyperparameter tuning in each domain separately, and then we selected the best performing models for the joint optimization.

The upper branch operating on the raw signal is inspired by EncodeNet [24] and uses consecutive residual blocks, which operate with high numbers of channels on the downscaled sample. The lower branch operating on spectral-domain is the SkipFCN [25], which connects the early low-level representations to latent layers to reduce over-fitting while stabilizing gradient flow.

More importantly, we wanted our research to give valuable feedback to doctors. Therefore, we inspected features that our trained AF detector has learned from samples provided by the Challenge to check whether these features were matching with ones recognized by professionals. These results are analyzed in the next section. For a more detailed description of the network architecture, see appendix A.

\section{RESULTS}

\subsection{Main Results}

Detecting the four categories: Normal, AF, Other and Noisy in terms of the official, F1 metric of the challenge, our proposed algorithm has scored $0.88,0.80,0.69,0.64$ points respectively, and 0.79 on average. The fact that our F1 score 0.64 on Noise detection was even higher then the same score of winning teams (see 2) shows that one is capable of reaching performance close to the state of the art methods without professional feature-engineering.

\subsection{Ambiguity of Annotations}

Using the website we designed for the alternative annotation, Expert-1 annotated 500 recordings. Comparing that annotation to the annotation of the cardiologists of the Challenge, we found that the two annotations showed matching only in $65 \%$ of cases, underlining the fact that classification of data set we worked on is challenging even for experts. Moreover, it turned out that considering only those recordings when both Expert-1 and the cardiologists of the challenge agreed on the classification (i.e., in case of the "evident recordings") the model also presented an almost identical (97.35\%) classification.

To visualize the overlap between the 3 annotations we also drew Venn diagrams, see Figure 2. We created diagrams for each class and marked with red the number of the samples

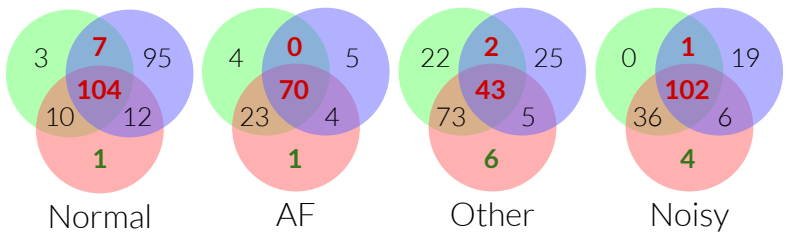

Figure 2: Venn diagrams showing the overlap between the 3 annotations: the official labels created by the cardiologists of the Challenge (green circle), Expert-1's annotation (blue circle) and the classification of the neural network (red circle).

that were unambiguous for cardiologists (i.e., the classification of Expert-1 and the cardiologists of the Challenge was the same). It is clearly visible that for the vast majority of these evident samples, the neural network predicted the same class as the cardiologists did. Additionally, only a tiny fraction of samples (their number is colored to green) were classified by our model in such a way that none of the cardiologists agrees with its prediction. Unfortunately, it is obvious that no statistical analysis is possible due to the low number of evaluated samples (500 compared to 8,528 available samples) and because only one expert completed that evaluation. However, these numbers strongly suggest that our results might have some medical relevance as our algorithm appears to mimic diagnosis of doctors.

\subsection{Confidence of The Classifier}

We created another web page to show recordings which were the easiest or the hardest to classify for our algorithm. To measure the "confidence" of the decision, we used the output of the last layer of our network (also called soft-max layer) which has three neurons and each neuron produces a number that correlates with the assumed probability of belonging to the normal, $A F$, or other classes, respectively. The fourth, noisy class is predicted by a separate network. We fed all recordings to the model and picked the top 10 recordings which produced the highest value from the neuron responsible for the normal class, and we repeated that experiment for the $A F$ class. We assumed that those recordings can also be interesting that were difficult to decide for our model, and therefore we selected the worse 10-10 recordings that resulted in the lowest value from the neurons of the normal and $A F$ class. The reason why we excluded other and noisy classes from examination are that these classes are only technically necessary for defining the problem, but they have no medical relevance regarding AF detection.

Then, we asked our experts to try to find some common features of the samples classified into the same classes by our model, and tell whether recordings classified "confidently" (i.e. with high output value) by our model were also evident for them. Similarly, we wanted to know whether they found the least "confidently" classified recordings obscure, too. They answered independently from each other, but their remarks were very similar in most aspects.

They both agreed that in the case of most confident predictions recordings had low noise contamination and this contamination could be easily distinguished from the signal. 
Also, they both mentioned that the main difficulty of classifying recordings classified with the lowest confidence was the high amplitude noise and the irregular baseline changes as it made P-wave detection very difficult. In case of hardly detectable $\mathrm{P}$-waves, they both would rather look at RR intervals to inspect whether they are regular or not.

In addition, Expert-2 noted that the model appeared to recognize the regularity of RR-intervals and used it as a strong evidence of normal class (doctors also consider it as a sign of healthy heart rhythm). Unfortunately, it was misleading sometimes because some recordings exhibited both unusually regular RR-intervals and also some clear signs of AF, and thus the model predicted normal class (with low confidence) instead of $A F$. Besides, Expert-1 noticed that all of the confidently classified AF recordings have arrhythmia absoluta (i.e., the RR intervals are always changing), and most of these recordings have high BPM value, while recordings of low confidence prediction have much lower BPM on average. She mentioned that arrhythmia absoluta and BPM are two of the most common features cardiologists are looking for in real-life clinical practice (along with the absence of $\mathrm{P}$-wave). Thus, she acknowledged that our model appeared to learne some medically relevant feature without explicitly programming to do so.

\subsection{Most relevant segments of recordings}

We divided the signal to $50 \mathrm{~ms}$ long segments and calculated the output of the neural network for each of them. As we did previously when we measured the "confidence" of the decision of the model, we took again the output of the three neurons of the soft-max layer, responsible for Normal, AF and Other classes, respectively. For each $50 \mathrm{~ms}$ long segment, the neuron that produced the highest value determined the color of the background behind the current section. When the neuron responsible for the normal class produced the highest value, then the background was colored to green. Similarly, the blue background indicated that the neuron of the other class had the highest output value, and red indicated atrial fibrillation. Additionally, higher values are translated to darker colors, so the darkness of background indicates the "confidence" of the prediction at a certain segment. An example of these graphs can be seen in Figure 3. By implementing that algorithm, we aimed to help doctors by highlighting the most relevant regions of ECG recordings. While our algorithm cannot substitute doctors, it might be a good tool to speed up the evaluation of long ECG recordings while unburdening physicians drawing their attention to the most important parts of the signal.

\subsection{Computational Complexity}

From the perspective of practical applicability in real-life medicine, our method is not just designed for classification, but performs well as a real-time detector by the nature of Fully Convolutional Networks: after the initial warm-up delay of $1.2 \mathrm{sec}$, we can generate new responses in less than 2 msec taking the last 20 second history into consideration. If the evaluation is centralized and we allow to compute responses in batches, the time required per sample is less than $0.5 \mathrm{msec}$.

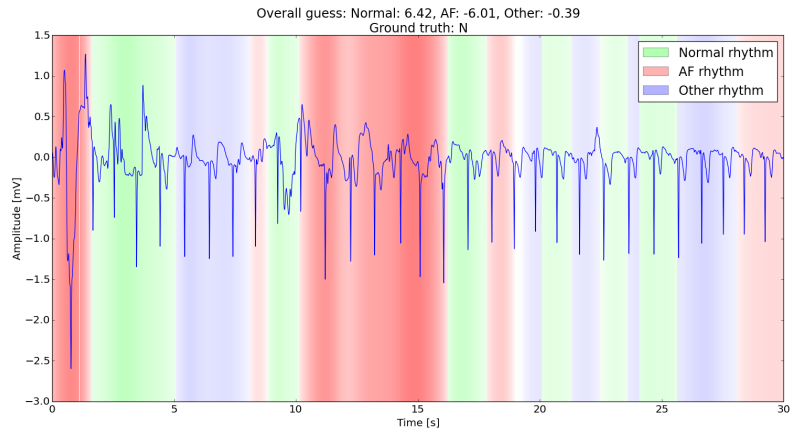

Figure 3: Example graph with colored background. Sections with green background are detected as normal rhythm sections, red indicates AF, and other arrhythmias are highlighted with blue.

\section{CONCLUSION}

While it is clear that current conditions demand expertise in both domains of cardiology and machine learning, the emergence of cheap hand-held devices creates a niche for approaches capable of utilizing larger amount of data, and that gives rise to adaptive and scalable algorithms, such as Deep Neural Networks.

We carried out an extensive architecture and hyperparameter search, and reported our findings on the Computing in Cardiology 2017 Challenge. To contribute to the field, we have open-sourced our project providing a general training environment for practitioners to quickly evaluate baseline performances on their dataset.

Our proposed algorithm provides visual reasoning and feedback for decision making that can significantly boost efficiency of AF detection in collaboration with experts. For deeper analysis of the performance, see appendix B. The website of our project is available at http://physionet. itk.ppke.hu/

Lastly, to help doctors to analyze long ECG recordings easily and quickly, we designed a tool that colors the background of the ECG plot highlighting the segments according to the prediction of the model.

\section{REFERENCES}

[1] C. A. Morillo, A. Banerjee, P. Perel, D. Wood, and X. Jouven, "Atrial fibrillation: the current epidemic," Journal of geriatric cardiology: $J G C$, vol. 14, no. 3, p. 195, 2017.

[2] B. B. Kelly, V. Fuster et al., Promoting cardiovascular health in the developing world: a critical challenge to achieve global health. National Academies Press, 2010.

[3] G. H. Tison, J. M. Sanchez, B. Ballinger, A. Singh, J. E. Olgin, M. J. Pletcher, E. Vittinghoff, E. S. Lee, S. M. Fan, and R. A. e. a. Gladstone, "Passive detection of atrial fibrillation using a commercially available smartwatch," JAMA Cardiology, vol. 3, no. 5, p. 409, 2018.

[4] S. P. Shashikumar, A. J. Shah, Q. Li, G. D. Clifford, and S. Nemati, "A deep learning approach to 
monitoring and detecting atrial fibrillation using wearable technology," 2017 IEEE EMBS International Conference on Biomedical $\&$ Health Informatics (BHI), 2017.

[5] "AF Classification from a short single lead ECG recording: the PhysioNet/Computing in Cardiology Challenge 2017." [Online]. Available: https://physionet.org/challenge/2017/

[6] 2019. [Online]. Available: https://store.alivecor.com/products/kardiamobile

[7] T. Teijeiro, C. A. García, D. Castro, and P. Félix, "Arrhythmia classification from the abductive interpretation of short single-lead ECG records," CoRR, vol. abs/1711.03892, 2017. [Online]. Available: http://arxiv.org/abs/1711.03892

[8] S. Datta, C. Puri, A. Mukherjee, R. Banerjee, A. D. Choudhury, R. Singh, A. Ukil, S. Bandyopadhyay, A. Pal, and S. Khandelwal, "Identifying normal, af and other abnormal ecg rhythms using a cascaded binary classifier," 2017 Computing in Cardiology (CinC), pp. 1-4, 2017.

[9] M. Zabihi, A. B. Rad, A. K. Katsaggelos, S. Kiranyaz, S. Narkilahti, and M. Gabbouj, "Detection of atrial fibrillation in ecg hand-held devices using a random forest classifier," in 2017 Computing in Cardiology (CinC), Sept 2017, pp. 1-4.

[10] S. Hong, M. Wu, Y. Zhou, Q. Wang, J. Shang, H. Li, and J. Xie, "Encase: An ensemble classifier for ecg classification using expert features and deep neural networks," in 2017 Computing in Cardiology (CinC), Sept 2017, pp. 1-4.

[11] K. He, X. Zhang, S. Ren, and J. Sun, "Deep Residual Learning for Image Recognition," arXiv:1512.03385 [cs], Dec. 2015, arXiv: 1512.03385. [Online]. Available: http://arxiv.org/abs/1512.03385

[12] S. Hochreiter and J. Schmidhuber, "Long short-term memory," Neural computation, vol. 9, pp. 1735-80, 12 1997.

[13] P. Warrick and M. N. Homsi, "Cardiac arrhythmia detection from ecg combining convolutional and long short-term memory networks," 2017 Computing in Cardiology Conference (CinC), 2017.

[14] F. Plesinger, P. Nejedly, I. Viscor, J. Halamek, and P. Jurak, "Automatic detection of atrial fibrillation and other arrhythmias in holter ecg recordings using rhythm features and neural networks," 2017 Computing in Cardiology Conference (CinC), 2017.

[15] M. Limam and F. Precioso, "Atrial fibrillation detection and ecg classification based on convolutional recurrent neural network," 2017 Computing in Cardiology Conference (CinC), 2017.

[16] M. Zihlmann, D. Perekrestenko, and M. Tschannen, "Convolutional recurrent neural networks for electrocardiogram classification," 2017 Computing in Cardiology Conference (CinC), 2017.

[17] F. Andreotti, O. Carr, M. A. F. Pimentel, A. Mahdi, and M. De Vos, "Comparing feature based classifiers and convolutional neural networks to detect arrhythmia from short segments of ecg," 2017 Computing in Cardiology Conference (CinC), 2017.

[18] S. Parvaneh, J. Rubin, R. Asif, B. Conroy, and S. Babaeizadeh, "Densely connected convolutional networks and signal quality analysis to detect atrial fibrillation using short single-lead ecg recordings," 2017 Computing in Cardiology Conference (CinC), 2017.

[19] Z. Xiong, M. Stiles, and J. Zhao, "Robust ecg signal classification for the detection of atrial fibrillation using novel neural networks," 2017 Computing in Cardiology Conference (CinC), 2017.

[20] D. Kingma and J. Ba, "Adam: A method for stochastic optimization," arXiv preprint arXiv:1412.6980, 2014. [Online]. Available: https://arxiv.org/abs/1412.6980

[21] G. Klambauer, T. Unterthiner, A. Mayr, and S. Hochreiter, "Self-Normalizing Neural Networks," arXiv:1706.02515 [cs, stat], Jun. 2017, arXiv: 1706.02515. [Online]. Available: http://arxiv.org/abs/1706.02515

[22] F. Yu and V. Koltun, "Multi-Scale Context Aggregation by Dilated Convolutions," arXiv:1511.07122 [cs], Nov. 2015, arXiv: 1511.07122. [Online]. Available: http://arxiv.org/abs/1511.07122

[23] K. He, X. Zhang, S. Ren, and J. Sun, "Deep residual learning for image recognition," in Proceedings of the IEEE Conference on Computer Vision and Pattern Recognition, 2016, pp. 770-778.

[24] L. A. Gatys, A. S. Ecker, M. Bethge, A. Hertzmann, and E. Shechtman, "Controlling perceptual factors in neural style transfer," in IEEE Conference on Computer Vision and Pattern Recognition (CVPR), 2017.

[25] J. Long, E. Shelhamer, and T. Darrell, "Fully convolutional networks for semantic segmentation," in Proceedings of the IEEE Conference on Computer Vision and Pattern Recognition, 2015, pp. 3431-3440. [Online]. Available: http://www.cv-foundation.org/ openaccess/content_cvpr_2015/html/Long_Fully_ Convolutional_Networks_2015_CVPR_paper.html

[26] A. v. d. Oord, S. Dieleman, H. Zen, K. Simonyan, O. Vinyals, A. Graves, N. Kalchbrenner, A. Senior, and K. Kavukcuoglu, "WaveNet: A Generative Model for Raw Audio," arXiv:1609.03499 [cs], Sep. 2016, arXiv: 1609.03499. [Online]. Available: http://arxiv.org/abs/1609.03499

[27] F. N. Iandola, M. W. Moskewicz, K. Ashraf, S. Han, W. J. Dally, and K. Keutzer, "Squeezenet: Alexnet-level accuracy with 50x fewer parameters and $<1 \mathrm{mb}$ model size," CoRR, vol. abs/1602.07360, 2016. [Online]. Available: http://arxiv.org/abs/1602.07360

[28] K. Simonyan and A. Zisserman, "Very Deep Convolutional Networks for Large-Scale Image Recognition," arXiv:1409.1556 [cs], Sep. 2014, arXiv: 1409.1556. [Online]. Available: http://arxiv.org/abs/1409.1556

[29] P. Rajpurkar, A. Y. Hannun, M. Haghpanahi, C. Bourn, and A. Y. Ng, "Cardiologist-Level Arrhythmia Detection with Convolutional Neural Networks," arXiv:1707.01836 [cs], Jul. 2017, arXiv: 1707.01836. [Online]. Available: http://arxiv.org/abs/1707.01836

[30] I. Goodfellow, Y. Bengio, and A. Courville, "Deep learning," 2016, book in preparation for MIT Press. [Online]. Available: http://www.deeplearningbook.org 


\section{APPENDIX}

\section{A. DETAILS OF THE NEURAL NETWORK}

\section{A.1 Activation Function}

We have found that one major reason of over-fitting where the network rather memorizes training samples than generalizes was the non-linearity applied after parametrized layers, the Rectified Linear Unit (ReLU): $\operatorname{relu}(x)=\max (0, x)$

The phenomenon of "dead neurons" is a well known and a frequent issue among networks that apply ReLU. Generally speaking, ReLU suppresses any inhibiting activation (by clipping off negative values), excluding a notable portion of neurons in the preceding layer from succeeding layers; thus, activation spectrum of the layer will be saturated. Practically it is acceptable if different samples cause different neurons to be mitigated; however, during train time some of the neurons can become completely silent since the gradient optimization prevents muted neurons to be trained (their gradient is 0 ). Because of this property, there is a considerable risk that numerous nodes will not be able to influence the response of classifier neurons.

Instead of ReLU, we used SELU activation function published in Self-Normalizing Neural Networks [21]. By changing the activation function, we were able to overcome the variance-problem of the networks applied, i.e. distance between training- and test-performance was reduced for identical architectures. For benchmarks on VGG models see Figure 4.

On the left side of Figure 4 two separate trends are revealed. Apparently, ReLU (bold lines) outperforms identical networks applied with SELU (light lines), almost reaching ideal performance. On the right side of Figure 4: We can see ReLU networks reaching their top test-performance in early stages of training, and by continuing their training their accuracy decreased. In contrast, the accuracy of SELU networks gradually improves throughout the entire training. Naming convention: ADAM stands for gradient optimization method, 16/19 for the number of layers that have adjustable weights, and double/halved/quart suffixes refers to the depth of each convolutional filter applied in corresponding VGG baseline networks.

\section{A.2 Dilated Convolution}

Receptive field problem was another obstacle we encountered while setting up the baselines experiments. Simply by changing the 2-dimensional convolutions ( $3 \times 3$ filters) to their 1-dimensional equivalent (1x9 filters), we ended up with a network that could barely cover multiple heartbeats. Since we have learned that atrial fibrillation can be episodic, it was essential extending search space of architectures that could cover entire episodes. By applying causal dilated convolutional filters used by [26], the receptive field was exponentially increased further improving our models' accuracy without involving variance problems (like max-pooling does) or sacrificing evaluation speed since applying dilated convolution results in minimal overhead compared to the traditional operation. For the visual example see Figure 5 .

\section{A.3 Spectrogram}

Representing a prerecorded signal to the frequency domain with Fast Fourier Transform is a favoured approach in the field of signal processing. Frequency analysis reveals each frequency band's presence in the signal, which may reveal periodic and aperiodic traits of the sample. In practice when a sample of the length $N$ is transformed with FFT, it produces two arrays of values of the same length $N$ representing complex (Im and Re) valued frequency coefficients. Usually, these two arrays are merged by the following formula $r=\sqrt{I m^{2}+R e^{2}}$ resulting in Power Spectrum (PS), while phase (being less informative about the signal) is omitted thus making the transformation irreversible. The problem with taking PS is that it discards temporal patterns (such as Q-T, R-R distance etc.) making convolutional layers useless. Furthermore, PS is not casual by design, meaning that the whole signal must be provided before obtaining PS. A frequently applied technique in speech recognition is taking multiple FFT of short overlapping windows sliding over input audio sample and concatenating short samples' PS into a multi-channel array. Another slight detail is to apply piece-wise natural logarithm on every element of the resulting array to increase the variance of signal and prevent strong frequencies repress ones which are weaker with orders of magnitude.

The main advantage of that method is that it preserves timedomain (temporal) patterns, while it reveals the presence of different frequencies in the signal. Furthermore, there is only a slight difference when we apply different weighting on internal values of the sliding window, while window size and stride (i.e. inverse degree of overlapping) heavily influences how long our resulting array will be and how many frequency bands will represent it (i.e. resolution of PS at given time instance). We have found it not just incredibly convenient, but also surprisingly effective to choose the highest possible degree of overlapping (window stridden by 1 ), and resampling resulting spectrogram to match the length of the original signal. Taking the original sample and redundant representation of ECG-recording (a 64 channel spectrogram) of the same length allowed us to apply two concurrent $\mathrm{NN}$ on each domain (temporal and spectral), and to concatenate resulting representations in-depth without being forced to reduce temporal dimension since both feature vectors were of the same length.

\section{A.4 Multi-Domain Representation Learning}

While both input spaces, temporal and spectral, had their challenges, we saw that - by designing preprocess steps for spectrogram training - the feature extractor network produced output of the same length as a time-domain equivalent algorithm. That led us to try and concatenate these pretrained feature extractors together to test whether multidomain representation could help the final layer to overcome issues specific to separate domain classification by complementing each other. Indeed, we found that the general behaviour of the time-domain network was as follows: increasing the accuracy of a single class at the expense of severe forgetting in other classes disappeared using multi-domain features. At the same time, spectral-domain networks struggled with variance problems, even with extremely low capacity. Also, networks trained in frequency domain were more dependent on the choice of training / evaluating data. These traits are omitted when feature extractors are working in 

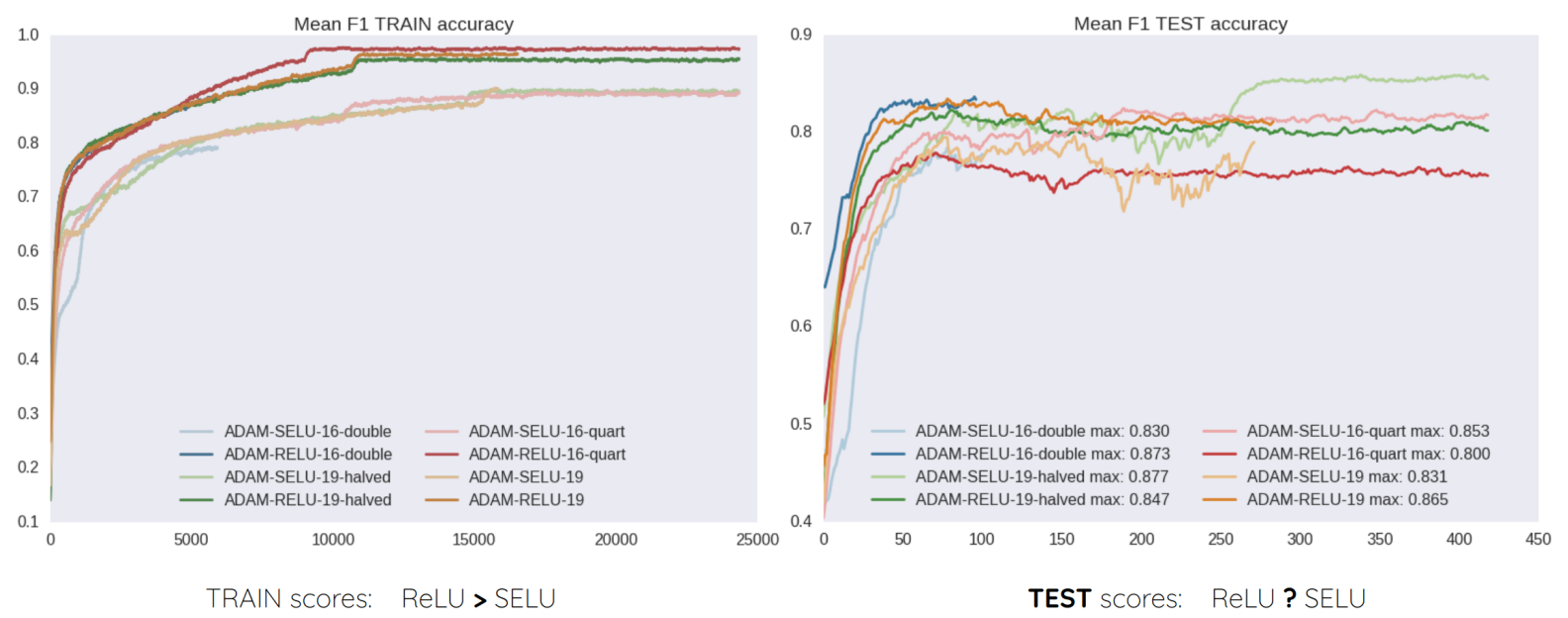

Figure 4: Mean F1 train accuracy

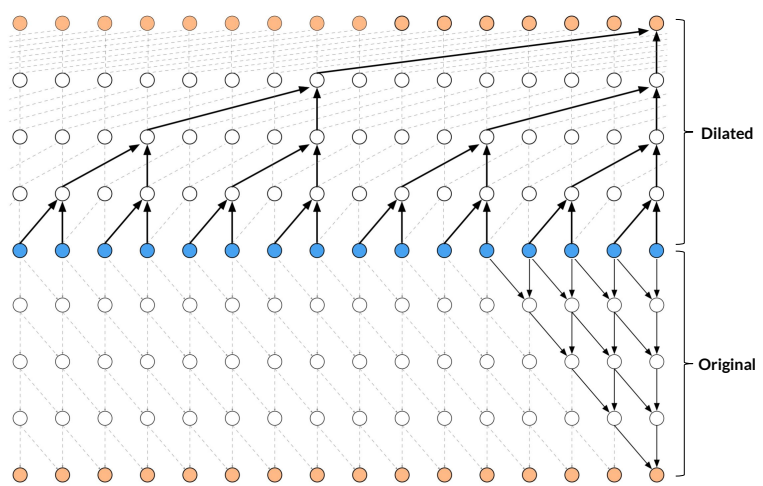

Figure 5: Using the same number of parameters at each node (2) with dilated convolutions, we can increase receptive field exponentially instead of linearly expanding receptive field of traditional convolutions

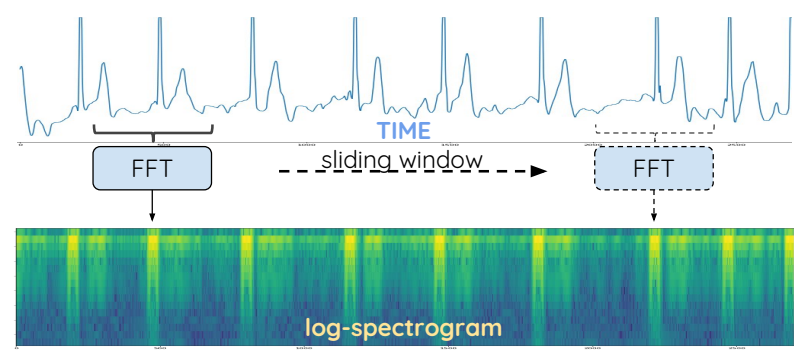

Figure 6: Using stride of 1 for 255 wide FFT windows resulted in almost identical length of original sample with 128 channels. In order to have a completely matching size in temporal dimension (horizontal axis), we resampled the log-spectrogram with nearest-neighbour interpolation. parallel as well. For a detailed description of our proposed model for CinC Challenge of 2017, see Figure 1.

\section{A.5 Training}

Each network was trained for 420 epochs on $80 \%$ of the challenge dataset, which we have resampled for each training instance. Depending on the complexity of the underlying architecture, training took 3-12 hours on a K80 GPU. Our proposed algorithm is designed in such a way that applied operations are completely causal; thus, the input can be processed on-line (also in real-time) without the presence of a complete sequence.

Inspired by their simplicity we have re-designed the classic ImageNet models: SqueezeNet v1.1 [27], VGG-16/19 [28], ResNet18/152 [23]. We also re-implemented a network proposed by Rajpurkar et al. [29], which was developed to perform well on a more balanced dataset of over sixty thousand single lead ECG samples, annotated by lead expert cardiologists. We reference this network in this writing as StanfordNet.

Deep representation learning algorithms tend to outperform humans when the network is shown more data than its opponent during its lifetime. While the problem itself could be a truly complex task from the perspective of traditional algorithmic solutions, it is less difficult with human references.

Referring to the rule of thumb mentioned in [30], it is increasingly evident that state-of-the-art supervised training methods can reach or even exceed human performance in general when 5000 samples are provided per class, and the overall dataset contains millions of training samples. That rule seems to apply to our case as well. For example, the dataset provided by the organizers of the Challenge contains over five thousand samples of healthy sinus-rhythm samples for which mean of test F1 scores are above 0.90, but considering that the whole training dataset contains only 8528 samples in total, it implies that getting deep neural networks to work requires a few workarounds. The usual recipe for training classifiers containing tens of millions of parameters 


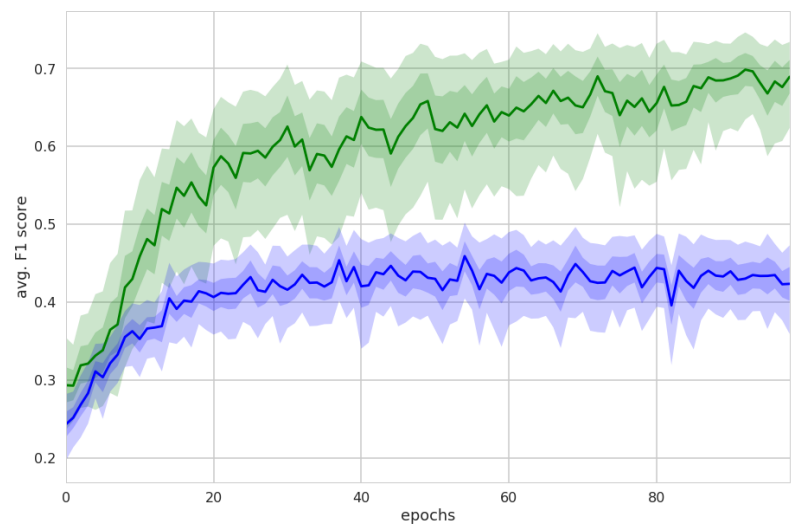

Figure 7: Results on running 18 different network architectures on a manually designed difficult training/test set separation (Blue), and on a random split in average (Green)

tells us that it is beneficial to find a model first that can be at least over-fitted on the training data, and then incrementally decrease degree of complexity by removing layers and shrinking the number of convolutional filters, while applying more and more regularization to avoid high variance. During the development phase we observed that by the time the network could not be improved further on the training set, it had already dropped in performance on the test set. Data preparation, training and evaluating scripts required to reproduce the following results are available at our open-source repository: https://github.com/botcs/itk-physionet-17

\section{B. PERFORMANCE ANALYSIS}

Instability. When we manually set random seeds for separating samples into training-test-evaluation classes, we came across a very interesting phenomenon: one can construct a train-evaluation set separation such that outcome can be extremely encouraging, over avg. $\mathrm{F} 1=0.9$ score, and for another choice, it could lead to much worse results, avg. $\mathrm{F} 1=0.5$, having every other detail of the training environment fixed. When we retrained 18 randomly chosen networks from our baseline evaluations on a training set which probably covered all problematic samples that may lie close to the decision boundary, independently of the underlying architecture results got better. Using exactly the same environment, with the same initialization of weights on a different choice of training samples, test performance seemed to have an upper bound - even our best-performing networks could not perform better than $F 1=0.6$. For comparison see Figure 7. In Figure 8, we analyze how sensitive is our most robust model: we do 10 -fold cross-validation, where each training is evaluated with two different seeds to eliminate the noise coming from random initializing the network.

18 networks initialized and optimized with the same fixed random seed on different training sets, which we have found randomly splitting the original dataset, reveals the greatest challenge in AF detection; namely, there is a tiny set of specific samples that is crucial for generalization.

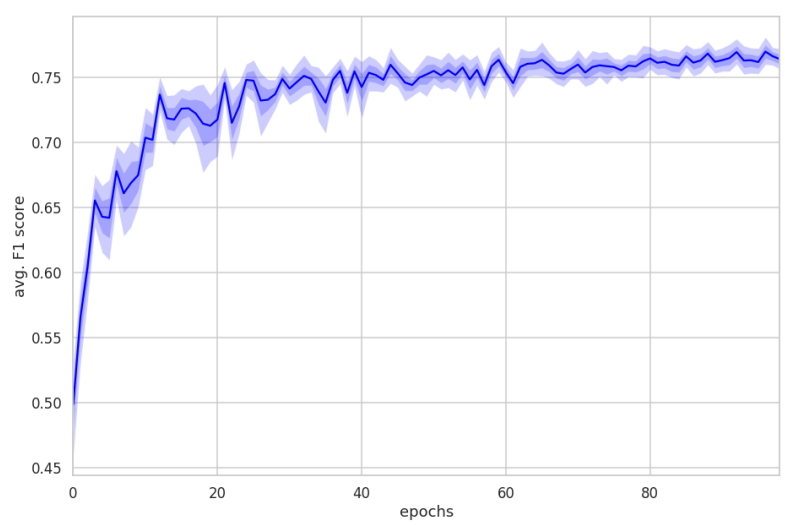

Figure 8: Test results computed from 10-fold cross validation using the best performing model.

Many competitors reportedly have overcome that issue by applying a common technique used in deep learning competitions: final prediction is not evaluated by a single network, but by ensembles of neural networks to make the algorithm more robust against the biased dataset. In groups where expert supervision of training data set was available, significant improvement was reported when ambiguous samples were removed from the already moderate-sized data set.

Note: The bands correspond to cover $99 \%$ and $68 \%$ of test scores and show that when certain training samples are missing, networks fail to generalize, and they are only able to reach a test score $\mathrm{F} 1=0.45$ with a small deviation, making it almost impossible to measure improvements on different architectures and optimization methods. At the same time, when essential training examples are available, we have space for experiments, shown by the increased width of the band.

\begin{tabular}{|c|c|c|c|c|c|c|c|c|c|c|c|}
\hline avg. F1 score & 0.70 & 0.72 & 0.73 & 0.72 & 0.76 & 0.78 & 0.74 & 0.74 & 0.75 & 0.81 & 0.73 \\
\hline SELU & & & $\checkmark$ & & & $\checkmark$ & & & $\checkmark$ & $\checkmark$ & $\checkmark$ \\
\hline Dilation & & $\checkmark$ & $\checkmark$ & & $\checkmark$ & $\checkmark$ & & $\checkmark$ & $\checkmark$ & $\checkmark$ & $\checkmark$ \\
\hline SqueezeNet 1.1 & $\checkmark$ & $\checkmark$ & $\checkmark$ & & & & & & & & \\
\hline VGG 19 & & & & $\checkmark$ & $\checkmark$ & $\checkmark$ & & & & & \\
\hline $\begin{array}{r}\text { StanfordECG } \\
\text { EncodeNet (ours) }\end{array}$ & & & & & & & $\checkmark$ & $\checkmark$ & $\checkmark$ & $\checkmark$ & \\
\hline SkipFCN (ours) & & & & & & & & & & & $\checkmark$ \\
\hline $\begin{array}{c}\text { Raw signal } \\
\text { Log-spectrogram }\end{array}$ & $\checkmark$ & $\checkmark$ & $\checkmark$ & $\checkmark$ & $\checkmark$ & $\checkmark$ & $\checkmark$ & $\checkmark$ & $\checkmark$ & $\checkmark$ & $\checkmark$ \\
\hline
\end{tabular}

Table 2: The average $F 1$ score results in time domain

\begin{tabular}{|c|c|c|c|c|c|c|c|c|c|c|c|}
\hline avg. F1 score & 0.65 & 0.63 & 0.63 & 0.69 & 0.74 & 0.75 & 0.53 & 0.51 & 0.54 & 0.68 & 0.79 \\
\hline SELU & & & $\checkmark$ & & & $\checkmark$ & & & $\checkmark$ & $\checkmark$ & $\checkmark$ \\
\hline Dilation & & $\checkmark$ & $\checkmark$ & & $\checkmark$ & $\checkmark$ & & $\checkmark$ & $\checkmark$ & $\checkmark$ & $\checkmark$ \\
\hline SqueezeNet 1.1 & $\checkmark$ & $\checkmark$ & $\checkmark$ & & & & & & & & \\
\hline $\begin{array}{r}\text { VGG } 19 \\
\text { StanfordECG }\end{array}$ & & & & $\checkmark$ & $\checkmark$ & $\checkmark$ & & & & & \\
\hline $\begin{array}{r}\text { StanfordECG } \\
\text { EncodeNet (ours) }\end{array}$ & & & & & & & $\checkmark$ & $\checkmark$ & $\checkmark$ & $\checkmark$ & \\
\hline SkipFCN (ours) & & & & & & & & & & & $\checkmark$ \\
\hline $\begin{array}{c}\text { Raw signal } \\
\text { Log-spectrogram }\end{array}$ & & & & & & & & & & & \\
\hline Log-spectrogram & $\checkmark$ & $\checkmark$ & $\checkmark$ & $\checkmark$ & $\checkmark$ & $\checkmark$ & $\checkmark$ & $\checkmark$ & $\checkmark$ & $\checkmark$ & $\checkmark$ \\
\hline
\end{tabular}

Table 3: The average $F 1$ score results in spectral domain

In Table 2 a summary of incremental improvements achieved on original time-series is visible. Among over 100 different alternative architectures, here we list the most revealing and instructive experiences of ours. 
In Table 3 the average F1 score results is visible in the spectral domain. Despite that different paths led us to the architecture of $S k i p F C N$, the main drive was to achieve comparable performance to time-domain models since input space was heavily redundant. For keeping our evaluations symmetric and providing a reference for evaluating identical architectures on the spectral domain, we list results of the same networks shown in Table 2 .

Computational requirements. The delay and complexity of our proposed model, depicted in Figure 1 is mainly characterized by the Fourier transform, and the complexity of the two parallel branches, working on time and frequency domain respectively. In total the model has fewer than 2 million trainable parameters, that takes up $8 \mathrm{MB}$ storage using 32-bit precision representation. We performed extensive time complexity measurements on the proposed model, using a single P100 Graphics Processor Unit. For the training, we used the mini-batch size of 64 with early-stopping after 100 epochs on the training data. On average, each training took 1 hour before reaching a peak of accuracy. In real-time operation (inference) using Fourier Transform window size of 255 , the model requires 354 measurement points to evaluate the first response. Based on the sampling rate of the recording device $(300 \mathrm{~Hz})$ that translates to a 1.18 s effective delay. Furthermore, we measured how fast the model evaluates a 20 s window. Without computing samples in parallel, the time-domain branch takes $1.33 \mathrm{~ms} \pm 821 \mathrm{~ns}$ and the frequency-domain branch takes $1.54 \mathrm{~ms} \pm 1.48 \mu$ s to extract features, in total the inference takes $1.68 \mathrm{~ms} \pm 2.11$ $\mu \mathrm{s}$. Using a batch size of 100 we can parallelize the computations with a sub-linear increase in time cost: $18.6 \mathrm{~ms} \pm$ $21.9 \mu$ s on time-domain, $32.1 \mathrm{~ms} \pm 127 \mu$ s on the frequency domain, $39.1 \mathrm{~ms} \pm 482 \mu$ s when branches are computed in parallel. 